\title{
Biotecnologia Applicata
}

\section{Generazione di strutture tissutali tridimensionali in vitro mediante l'uso di cellule staminali}

\section{Lombardi}

Dottore in Biotecnologie Medico-Diagnostiche, Studente presso il Corso Magistrale in Terapie Biologiche e Cellulari in Medicina, CdL in Biotecnologie Mediche, Medicina, Università degli Studi di Firenze. Tirocinante del Laboratorio Interdipartimentale di Nefrologia Cellulare e Molecolare diretto dalla Professoressa P. Romagnani, Facoltà di Medicina e Chirurgia, Università degli Studi di Firenze

In Vitro generation of three dimensional structures employing Stem Cells

ABstract. The development of new knowledge on stem cells, and the contemporary advancement of the techniques based on tissue engineering, allow nowadays to enlarge the horizons of regenerative medicine and of its application in therapeutic field. In this paper are quoted the techniques and the basic possibilities concerning the de novo creation of tissues and organs in vitro, pointing out both benefits, and the most complicated aspects which make this approach still barelyfeasible in therapy, as the problem related to the immune rejection. Is merging the thematic of immune rejection and of tissue engineering that is introduced a possible alternative for the creation of renal structures totally made in vitro, but also totally functional.

KeY Words. Tissue engineering, Stem Cells, Scaffold, Therapeutic Cloning

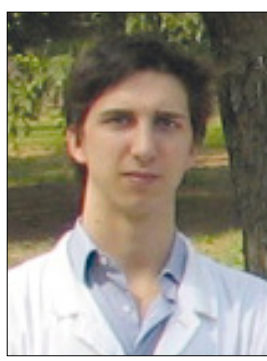

Duccio Lombardi

\section{Introduzione}

La generazione di strutture tissutali tridimensionali in vitro si basa su quella che è l'ingegneria tissutale ed il relativo impiego in tale ambito di cellule staminali. L'obiettivo che si propone è quello di creare organi o tessuti impiantabili e pronti per la sostituzione sia in caso di insulto acuto,

che di danno cronico.

Con il termine ingegneria tissutale ci si riferisce a tutti quegli approcci aventi finalità di riparare, o anche rimpiazzare, tessuti colpiti da patologie tra le più varie: congenite, croniche, tumorali, da insulto o derivanti da infezioni. In particolare i tessuti possono essere ingegnerizzati in vivo, qualora si stimoli il corpo all'autorigenerazione mediante l'impiego di appositi biomateriali, oppure ex vivo: le cellule sono in tal caso espanse in coltura, montate su apposite impalcature (scaffold), quindi rese al paziente.
Per realizzare "organi in provetta" a fini di trapianto è però necessario avere una risorsa rinnovabile di cellule, quali sono le cellule staminali, ed in particolar modo le cellule staminali embrionali (ESC). Queste ultime sono capaci di differenziare verso tutti i tipi cellulari e di proliferare virtualmente a tempo indeterminato. Ciò nonostante l'impiego di ESC nell'ingegneria tissutale al momento deve superare alcune sfide come quelle relative alle metodologie dirette ad un corretto differenziamento e alla prevenzione della formazione di tumori derivanti dal loro utilizzo. Si deve inoltre prevenire il rigetto immunitario delle stesse ESC, ma anche ottimizzare le proprietà degli scaffold relativamente alle caratteristiche di queste cellule.

Tali problematiche non si manifestano nel caso di impiego di cellule staminali adulte (ASC). Le Adult Stem Cells difatti non presentano potenziale teratogeno, sono già parzialmente differenziate verso le linee cellulari del tessuto di origine ed inoltre possono essere espiantate, coltivate, espanse e ri-fornite al paziente totalmente in autologo, evitando così il rischio di rigetto. Queste 
cellule hanno però una capacità replicativa limitata: sono perciò ottenibili un minor numero di cellule per l'impiego terapeutico. Inoltre presentano una ristretta plasticità differenziativa, che non è altresì riscontrata nel caso delle ESC.

Quel che è maggiormente importante considerare è che l'utilizzo di prodotti derivanti da ingegneria tissutale fornirebbe una terapia che, da un lato aumenterebbe notevolmente la qualità di vita del paziente a cui sarebbe fornito un trattamento a più ampio raggio, dall'altro ridurrebbe notevolmente l'ospedalizzazione dei pazienti, abbassando quindi i relativi costi sociosanitari (1).

\section{Ingegnerizzazione tissutale}

Esistono essenzialmente tre approcci per la generazione di tessuti ingegnerizzati (2):

$\mathrm{Si}$ possono impiegare cellule isolate per rimpiazzare la componente cellulare depleta.

È possibile usare materiali acellulari capaci di indurre la rigenerazione tissutale; esempio sono le spugne di matrigel imbevute con fattori pro-angiogenici al fine di favorire la neoangiogenesi.

Altra possibilità è quella dell'impiego di una combinazione di cellule e materiali acellulari, tipicamente sotto forma di scaffold.

\section{Cellule isolate}

Dette anche "cellule di sostituzione", il loro impiego è stato a lungo l'approccio maggiormente utilizzato per la sostituzione cellulare. In tal caso sono fornite soluzioni cellulari al paziente, le quali spontaneamente andranno a migrare nella sede di appartenenza o di danno. La prima applicazione storica di tale tecnica risale al trapianto di midollo osseo, evento che permette alle cellule staminali ematopoietiche del donatore di ripopolare il midollo dell'ospite e, di conseguenza, la sua componente ematica (3). Questi precursori ematopoietici è stato in seguito dimostrato che possono originare progenitori endoteliali utili per la neoangiogenesi in tessuti ischemici $(4,5)$, così come possono differenziare, e quindi rigenerare, ossa, cartilagine, miociti (6) o migrare nel SNC per produrre neuroni $(7,8)$.

I prodotti d'ingegneria tissutale basati su cellule di sostituzione trovano largo impiego nel caso di sostituzione epidermica o anche nel caso di rigenerazione ossea e cartilaginea mediante cellule staminali mesenchimali (9).

\section{Combinazione di cellule e materiali}

Gli approcci di ingegneria tissutale che si basano sull'impiego di scaffold e cellule possono essere suddivisi in due categorie in base a quella che è l'esposizione delle cellule al sistema immunitario dell'ospite una volta impiantati (10).

\section{Open System}

Le cellule sono immobilizzate su di uno scaffold tridimensionale altamente poroso costituito da materiali sintetici, naturali o da un composito di entrambi (2,11-13).

Idealmente lo scaffold deve fornire il microambiente ottimale per la crescita cellulare, pertanto dovrà possedere ottime proprietà di trasporto dei nutrienti e di $\mathrm{O}_{2}$, buona integrità meccanica ed una degradazione temporalmente controllata. Lo scaffold oltre a fornire un ambiente tridimensionale di sostegno, mette in contatto le cellule permettendogli di "auto-assemblarsi" e di formare i vari componenti caratterizzanti un dato microenvironment tissutale. E importante che il materiale di cui è costituito lo scaffold si degradi progressivamente all'aumentare della deposizione di matrice extracellulare da parte delle cellule sedimentate in esso. A tal fine sono impiegati biomateriali sintetici degradabili (come il PLGA [14]) o materiali naturali (come collageno [15], idrossiapatite [16] o calcio carbonato [17]). I materiali naturali presentano caratteristiche che assicurano una migliore adesione cellulare, di contro i materiali sintetici hanno proprietà che sono meglio controllabili, come il rate di degradazione, le proprietà meccaniche e la porosità (2).

Potendo tali polimeri fungere da supporto fisico, risultano sistemi molto promettenti per permettere la formazione di strutture tissutali tridimensionali complesse. Lo scaffold fornisce difatti vincoli fisici per la diffusione e l'orientamento delle cellule, nonché pori che garantiscono uno spazio adeguato per il rimodellamento della struttura tissutale. Il suo ruolo è quindi, idealmente, quello di guidare il differenziamento nel tipo cellulare desiderato facendo leva sui vincoli che impone alle cellule stesse.

Il vantaggio di tale sistema risiede ovviamente nel fatto che, mentre si specializzano, le cellule si "auto-assemblano" dando quindi luogo ad un pattern differenziativo che mima la differenziazione cui si assiste durante embriogenesi e sviluppo fetale, soprattutto qualora siano impiegate ESC. Ciò permette di indirizzare molto bene verso uno specifico lineage cellulare.

L'uso di sistemi aperti ha riportato successi nella creazione di molti sostituti biologici come ossa (18), cartilagine (19), cardiomiociti (20), retina (21) e pancreas (22). 


\section{Closed System}

Anche in tal caso le cellule sono posizionate su di una matrice con funzione di impalcatura ove differenziano, ma la finalità del sistema chiuso è piuttosto quella di immobilizzare le cellule in matrici che fungano da barriera per i componenti immunologici dell'ospite, così da evitare il rischio di rigetto.

Le cellule sono quindi fermate su membrane semipermeabili: permeabili ad $\mathrm{O}_{2}$ e nutrienti, ma non a cellule immunitarie, immunoglobuline o frazioni del complemento $(10,11)$.

Inoltre i sistemi chiusi proteggono l'ospite dal rischio tumorigenico associato all'impiego di ESC grazie proprio alla funzione di barriera che tale scaffold assume.

Questo tipo di sistema è molto impiegato nel trattamento del diabete (23), nell'insufficienza epatica (24) e nel Parkinson (25).

Basandosi l'ingegneria tissutale principalmente sull'utilizzo di ESC, vengono ad evidenziarsi non solo il problema etico relativo al loro impiego, ma anche quello inerente il rigetto di tali cellule non autologhe, come già evidenziato parlando dei closed system.

Una soluzione per queste due problematiche potrebbe risiedere nel therapeutic cloning, ossia nel trasferimento del nucleo di una cellula somatica terminalmente differenziata, in un oocita previamente denucleato: ciò porta alla riprogrammazione del nucleo del donatore e alla formazione di cellule staminali embrionali. Tali cellule, possedendo il genoma del donatore, potranno essere impiegate totalmente in autologo evitando il rischio e $\mathrm{i}$ problemi connessi al rigetto immunologico. Inoltre questa tecnica potrebbe teoricamente fornire una risorsa illimitata di cellule per terapie rigenerative, in quanto la capacità replicativa delle Embryonic Stem Cells è virtualmente illimitata.

Nello studio riportato (26), cellule derivate da therapeutic clonic sono state usate per creare unità renali funzionali, le quali si sono auto-organizzate in strutture simil-glomerulari e simil-tubulari che hanno prodotto un liquido analogo all'urina.

In tale paper è stato inoltre provato che tale metodica permette di evitare ed eliminare la risposta immunitaria associata ai trapianti, e che potrebbe quindi potenzialmente prevenire la necessità di impiego di terapie immunosoppressive, le quali comportano gravi rischi di complicazioni infettive, neoplastiche ed autoimmunitarie (27).

Le cellule renali clonate possedevano il DNA nucleare di un fibroblasto derivante dal toro donatore, mentre il DNA mitocondriale apparteneva all'oocita in cui è stato impiantato il nucleo. L'unica componente che quindi potrebbe risultare immunogena è rappresentata dalle proteine mi-

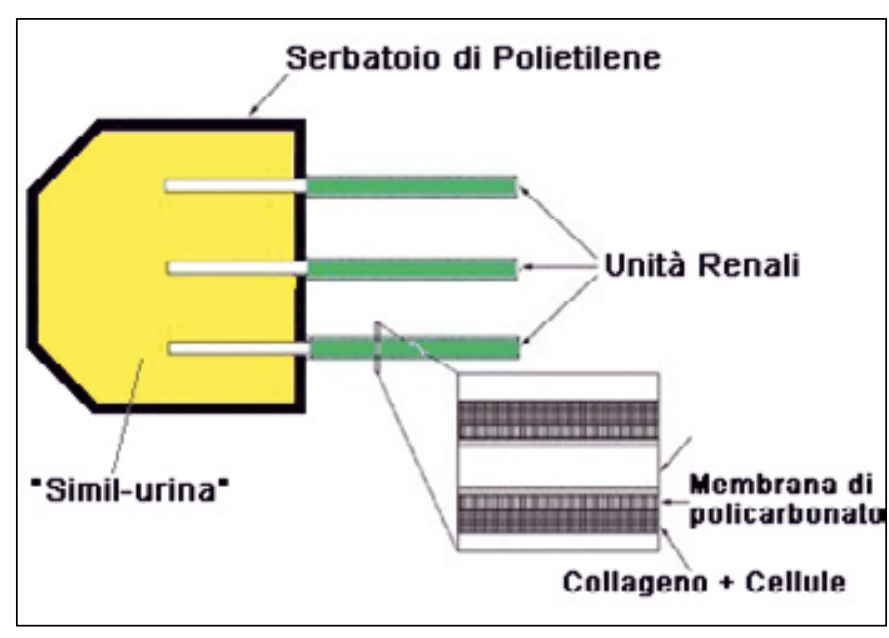

Fig. 1 - Rappresentazione schematica di un'unità funzionale renale, struttura poi impiantata sottocute nel toro donatore del nucleo.

tocondriali, le quali non sono ovviamente self, bensì derivano dall'oocita denucleato. Ciò nonostante le cellule clonate e poi differenziate sia in senso cardiomiocitario, sia miocitario liscio, sia renale, non sono state rigettate $\mathrm{e}$ sono rimaste vitali per un lungo periodo dopo l'impianto nell'animale da cui era stato prelevato il nucleo.

Gli autori hanno perciò dimostrato la possibilità di una ricostituzione e di un rimodellamento strutturale del tessuto renale a partire da cellule renali derivate da un feto bovino clonato.

Le cellule renali sono state isolate da un metanefro clonato di 56 giorni, e dopo essere state espanse in vitro, sono state sedimentate su quella che è stata chiamata "unità renale". Tale unità consta di tre strutture cilindriche di policarbonato rivestite di collageno che confluiscono in un sistema di raccolta di fluidi (Fig. 1). Le cellule erano in particolare poste sulle tre strutture cilindriche.

L'unità renale è stata quindi impiantata sottocute nell'animale da cui era stato preso il nucleo cellulare, in quello che è dunque un trapianto autologo. Espiantata dopo 12 settimane è stato possibile notare che le cellule si erano organizzate in tali strutture simil-glomerulari e similtubulari (Fig. 2) e che inoltre possedevano la capacità di escrezione unidirezionale di prodotti metabolici di scarto, come evidenziato dalla presenza di un liquido di color giallo paglia molto simile ad urina riscontrabile nella sacca di raccolta.

La fenotipizzazione in vitro di queste cellule ha confermato l'espressione di proteine specifiche renali tra cui la sinaptopodina, aquaporina-1, aquaporina-2 e la proteina di Tamm-Horsfall.

Le cellule renali erano inoltre capaci di produrre sia 


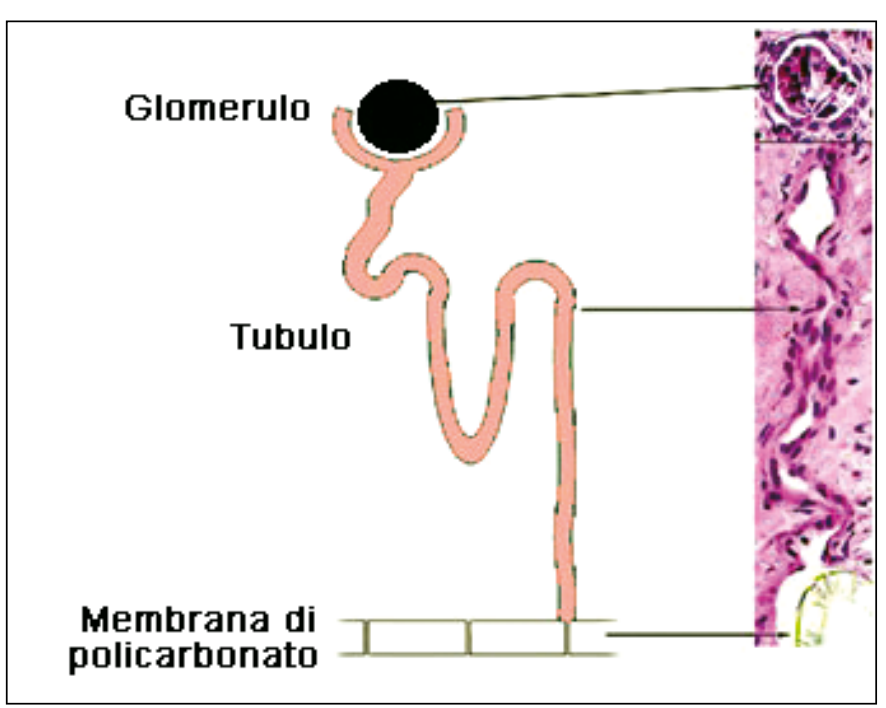

Fig. 2 - Organizzazione simil-nefronica delle cellule derivate dal metanefro bovino. $\mathbf{E}$ possibile in particolare notare la formazione di una struttura simil-glomerulare e di una simil-tubulare strettamente connesse tra loro e confluenti nella membrana di policarbonato.

eritropoietina che la forma attiva della vitamina D3, ma soprattutto erano capaci di filtrazione, riassorbimento e funzioni secretorie unidirezionali. Il glucosio, fisiologicamente riassorbito a livello del tubulo prossimale, non era presente nel fluido che presentava però un $\mathrm{pH}$ pari a 8.1.

Da notare che le unità renali espiantate presentavano un'intensa vascolarizzazione e che, durante il processo di auto assemblaggio in strutture simil-nefroniche, il tessuto renale si era integralmente connesso in modo unidirezionale alla sacca di raccolta. Era inoltre possibile evidenziare una chiara continuità tra i glomeruli maturi, i tubuli in cui essi sfociano e la membrana di policarbonato.

Anticorpi diretti contro acquaporina-1, acquaporina-2 e sinaptopodina hanno permesso di identificare rispettivamente segmenti tubulari dei dotti collettori e glomerulari. Al contrario nei controlli negativi effettuati mediante l'impiego di cellule con nucleo allogenico non si aveva né la presenza di tali , né era prodotto alcun liquido simile all'urina, ma soprattutto era possibile notare un esteso processo di necrosi compatibile con il quadro di un rigetto acuto. Il rigetto non era invece rilevabile nel caso di impiego di cellule autologhe, come dimostrato sia mediante test da ipersensibilità ritardata in vivo, che da analisi di produzione di IFN $\gamma$ dopo stimolazione in vitro dei Linfociti T dell'animale. Il DNA allogenico mitocondriale non induce quindi rigetto nelle unità trapiantate con cellule di derivazione autologa.

\section{Conclusioni}

Le nuove tecnologie basate sull'impiego di ingegneria tissutale e di impalcature che consentano il corretto differenziamento delle cellule staminali sedimentate su di esse appare di grande interesse nel campo della medicina rigenerativa, e potrebbe garantire ottime prospettive a lungo raggio nell'approccio e nel trattamento non solo di patologie di natura cronica, ma anche nell'intervento in seguito a danni d'organo acuti. In particolare la capacità di bioingegnerizzare cellule staminali primordiali in strutture funzionali molto più complesse potrebbe far superare quelli che sono i due principali problemi nella medicina del trapianto: il rigetto immunitario e la carenza di organi disponibili, come è stato ampiamente dimostrato nell'articolo di Lanza et al (26).

\section{Riassunto}

Lo sviluppo di sempre nuove conoscenze sulle cellule staminali e il contemporaneo progresso delle tecniche basate sull'ingegneria tissutale permettono, ad oggi, di ampliare gli orizzonti della medicina rigenerativa e le sue applicazioni in ambito terapeutico. In questo articolo sono riportate le tecniche e le possibilità di base inerenti la creazione de novo di tessuti ed organi in vitro, evidenziandone sia i benefici, sia le problematiche di maggior rilievo che rendono tali approcci ancora poco applicabili in terapia, come la questione correlata al rigetto di natura immunitaria. Unendo la tematica del rigetto autoimmune e dell'ingegneria tissutale sarà quindi introdotta una possibile alternativa per la creazione di strutture renali totalmente create in vitro, ma anche totalmente funzionali.

Parole Chiave. Ingegneria Tissutale, Cellule Staminali, Scaffold, Therapeutic Cloning.

\author{
Indirizzo degli Autori: \\ Duccio Lombardi \\ Via B. Scala 23 \\ 50126 Firenze \\ lombarduccio@alice.it
}




\section{Bibliografia}

1. Lanza R. Gearhart J. Hogan B. Melton D. Pedersen R. Thomson J. West M. Handbook of Stem Cells. Amsterdam: Elsevier Inc Academic Press, 2004; 737-42.

2. Langer R, and Vacanti JP. Tissue engineering. Science 1993; 260: 920-6.

3. Till JE, and McCulloch EA. Hemopoietic stem cell differentiation. Biochim Biophys Acta 1980; 605: 431-59.

4. Walter DH, and Dimmeler S. Endothelial progenitor cells: regulation and contribution to adult neovascularization. Herz 2002; 27: 579-88.

5. Edelberg JM, Tang L, Hattori K, Lyden D, and Rafii S. Young adult bone marrow-derived endothelial precursor cells restore aging-impaired cardiac angiogenic function. Circ Res 2002; 90: e89-e93.

6. Pittenger MF, Mackay AM, Beck SC, et al. Multilineage potential of adult human mesenchymal stem cells. Science 1999; 284: 143-7.

7. Brazelton TR, Rossi FM, Keshet GI, and Blau HM. From marrow to brain: expression of neuronal phenotypes in adult mice. Science 2000; 290: 1775-9.

8. Mezey E, Chandross KJ, Harta G, Maki RA, and McKercher SR. Turning blood into brain: Cells bearing neuronal antigens generated in vivo from bone marrow. Science 2000; 290: 1779-82.

9. Bruder SP, Jaiswal N, Ricalton NS, Mosca JD, Kraus KH, and Kadiyala S. Mesenchymal stem cells in osteobiology and applied bone regeneration. Clin Orthop 1998; 355(Suppl.): S247-S256.

10. Uludag H, De Vos P, and Tresco PA. Technology of mammalian cell encapsulation. Adv Drug Deliv Rev 2000; 42: 29-64.

11. Vacanti JP, and Langer R. Tissue engineering: the design and fabrication of living replacement devices for surgical reconstruction and transplantation. Lancet 1999; 354(Suppl. 1): SI32-SI34.

12. Lanza RP, Langer RS, and Chick WL. Principles of tissues engineering. Academic Press/R.G. Landes, San Diego, Austin, 1997.

13. Langer RS, and Vacanti JP. Tissue engineering: the challenges ahead. Sci Am 1999; 280: 86-9.

14. Mooney DJ, Baldwin DF, Suh NP, Vacanti JP, and Langer R. Novel approach to fabricate porous sponges of poly (D, L-lactic-co-glycolic acid) without the use of organic solvents. Biomaterials 1996; 17: 1417-22.
15. Chevallay B, and Herbage D. Collagen-based biomaterials as 3D scaffold for cell cultures: applications for tissue engineering and gene therapy. Med Biol Eng Comput 2000; 38: 211-8.

16. Friedman CD, and Costantino PD. Hydroxyapatite cement, a smart biomaterial for craniofacial skeletal tissue engineering. Surg Technol Int 1998; 7: 421-3.

17. Kreklau B, Sittinger M, Mensing MB, et al. Tissue engineering of biphasic joint cartilage transplants. Biomaterials 1999; 20: 1743-9.

18. Ohgushi H, Miyake J, and Tateishi T. Mesenchymal stem cells and bioceramics: strategies to regenerate the skeleton. Novartis Found Symp 2003; 249: 118-27; discussion 127-32, 170-4, and 239-41.

19. Sherwood JK, Riley SL, Palazzolo R, et al. A three-dimensional osteochondral composite scaffold for articular cartilage repair. Biomaterials 2002; 23: 4739-51.

20. Shinoka T, Shum-Tim D, Ma PX, et al. Creation of viable pulmonary artery autografts through tissue engineering. J Thorac Cardiovasc Surg 1998; 115: 536-45; discussion 545-6.

21. Lu L, Yaszemski MJ, and Mikos AG. Retinal pigment epithelium engineering using synthetic biodegradable polymers. Biomaterials 2001; 22: 3345-55.

22. Hasirci V, Berthiaume F, Bondre SP, et al. Expression of liver-functions by rat hepatocytes seeded in treated poly (lactic-coglycolic) acid biodegradable foams. Tissue Eng 2001; 7: 385-94.

23. Sefton MV, May MH, Lahooti S, and Babensee JE. Making microencapsulation work: conformal coating, immobilization gels, and in vivo performance. J Control Release 2000; 65: 173-86.

24. Uludag H, and Sefton MV. Microencapsulated human hepatoma (HepG2) cells: in vitro growth and protein release. J Biomed Mater Res 1993; 27: 1213-24.

25. Wang Y, Wang SD, Lin SZ, et al. Transplantation of microencapsulated PC12 cells provides long-term improvement of dopaminergic functions. Chin J Physiol 1997; 40: 121-9.

26. Lanza RP, Chung HY, Yoo JJ, et al. Generation of histocompatible tissues using nuclear transplantation. Nature Biotechnology 2002; 20: 689-96.

27. Lanza RP, Cibelli JB, and West MD. Prospects for the use of nuclear transfer in human transplantation. Nat Biotechnol 1999; 17: 1171-4. 